\title{
La Cámara de Comercio de Sevilla. Entidad empresarial y proyección política
}

\section{ENCARNACION LEMUS LOPEZ}

\section{EL MARCO DE ESTA INVESTIGACION; SUS OBJETIVOS}

La presente comunicación se inscribe dentro de un trabajo de investigación con el que se busca abordar el estudio pormenorizado de la Cámara de Comercio, Industria y Navegación como institución' inserta en el decurso político y económico de Sevilla en el primer tercio del siglo XX. Con este objetivo, acudimos desde una perspectiva singular a recomponer una nueva faceta de la Restauración en Sevilla. En este campo, los trabajos que sobre la Política y los políticos se han desarrollado, o están en transcurso, bajo la dirección del profesor Sánchez Mantero -de cuyos resultados se da cuenta en este Congreso- han abordado sistemáticamente la secuencia completa de la política de partidos. Complementariamente, por parte de los profesores Macarro Vera y González Fernández se nos viene demostrando el desarrollo en la organización y actividades del sector obrero. En este marco, con el estudio de la Cámara queremos incluir una nueva secuencia centrada en el empresariado.

Con los precedentes citados, no iniciamos el tema desde el vacío, por el contrario, lo hacemos desde una posición definida, asumiendo: primero la base económica del poder político $\mathrm{y}$, asimismo, el exiguo carácter de la oligarquía en la provincia de Sevilla, tal como se afirma en los trabajos de Sierra Alonso y Álvarez Rey, respectivamente (1). Con tal convencimiento, esperamos que el conocimiento de la actividad de la Cámara ilustre convenientemente esa fusión anunciada entre la tarea política y la tarea económica de una escasa oligarquía.

(1) SIERRA ALONSO, M. "Empresarios y políticos en la Sevilla de la Restauración: la familia Ybarra". Espacio, Tiempo y Forma, serie y Historia Contemporánea, t. 3, 1990, pp. 143-158, y ALVAREZ REV, L. "Elites políticas en Sevilla durante la crisis de la Restauración (1898-1931)", item, pp. 213-228. 
Si éste es el objetivo general, en nuestro acercamiento a la institución se irá desgranando de forma paulatina. Así, inicialmente habrá que trazar la progresiva composición de la Directiva, para con ello interrogarnos: primero, sobre la existencia o no de un grupo o grupos coherentes que hagan notar su predominio y, segundo, sobre si la naturaleza de esos grupos es básicamente económica o política.

A continuación, es necesario examinar las actitudes que la Cámara manifiesta en momentos clave de la política nacional -la crisis finisecular, la crisis de 1913, el año 1917, el golpe de Estado de Primo de Rivera y la caída de su dictadura, la proclamación de la República y su evolución. Con estos análisis será posible detectar si se producen distintas orientaciones entre grupos diversos dentro de la Cámara.

Por otra parte, es también nuestra intención identificar qué función corresponde a esta institución en la evolución económica de estos años y qué actividades económicas recibieron su preferente atención.

Estos planteamientos constituyen el marco sobre el que se inscribe la presente comunicación, centrada en un pormenorizado análisis del funcionamiento de la Cámara en la inflexión de 1917. En este sentido, incluimos previamente una breve referencia a la organización de la Cámara con anterioridad a ese momento y, seguidamente, nos detenemos en establecer conexiones entre su composición y su actividad en la citada fecha.

\section{LA CAMARA ANTES DE 1917}

El surgimiento de la Cámara de Comercio, Industria y Navegación de Sevilla en junio de 1886 se encuentra naturalmente unida al real decreto de ese año (Gaceta de 12 abril), que establecía como obligatoria la formación de un organismo de esta naturaleza al menos en todas las capitales de provincia. Con ello, se daba fin a un período de vacío que había seguido a la desaparición de las instituciones que en el Antiguo Régimen se habían ocupado del fomento y de la organización en tales materias (por ejemplo, las Juntas de Comercio). Desde el primer momento, aparece simultáneamente definido su doble carácter: de un lado, su sentido de organismo público de carácter consultivo; de otro, su esencia como corporación en defensa de los intereses concretos de sus asociados. Entre estas dos naturalezas se ha desenvuelto la historia de las Cámaras y ambas asoman en el ejemplo sevillano.

Con el carácter se precisan también sus funciones, les correspondía: a) coordinarse con la Administración para proponerle realizacio- 
nes o solicitarle reformas en favor del progreso económico; b) servir como órgano consultivo de los gobiernos; c) promover el desarrollo económico; d) promover la uniformidad en las prácticas mercantiles; d) fomentar la enseñanza en materias de economía; f) actuar de mediadora en conflictos surgidos entre los sectores productivos; g) colaborar en la inspección de las actividades económicas.

Hecha pública esta normativa, el 5 de mayo, por nombramiento directo del gobernador civil, se formó una comisión organizadora, tras cuyas gestiones pudo constituirse definitivamente la Cámara en Sevilla el 13 de junio, sin haber chocado con divergencias graves de intereses entre los diferentes sectores económicos (2). La nueva corporación representaba en ese momento a 342 socios, de quienes se acordó recaudar, en concepto de cuota, 905 pesetas al mes para su sostenimiento.

Encontramos en la presidencia general de la institución a José Montes Sierra, con él comparten la directiva: Andrés Fariña como presidente de la sección de Comercio; Saturnino Fernández y Fernández Industria- y Gonzalo Segovia Ardizene -Navegación-; junto a Carlos Lacave de secretario; Ricardo Santaló de contador y Francisco Ysern Maury (3). Dos notas importantes en relación con el nombramiento: primero, la intervención inicial simbólica del republicanismo de Montes Sierra. Decimos simbólica, porque, en realidad, su presencia hay que ponerla en relación con una preeminencia del sector naviero. Segundo, que las mayores firmas sevillanas, Hijos de M. Fernández Palacios, Pando Rodríguez y Cía., hijos de Luca de Tena, Mensaque Rodríguez y Cía, Ybarra..., aunque integradas, no se situaron a la cabeza de la iniciativa.

En 1911, con la publicación de la decisiva ley de Bases (Gaceta 1 de junio), se inaugura la etapa de apogeo en la historia de las Cámaras de Comercio -es forzoso distinguir entre la trayectoria anterior y posterior a 1911. Con la ley de Bases las cámaras son definitivamente definidas

(2) Hago hincapié en ello, puesto que no fue esa la actitud predominante en todas las ocasiones. Por ejemplo, en el caso de Alicante, se ha puesto de manifiesto el choque inicial entre los intereses de los grupos comerciales y financieros con los específicamente industriales. VIDAL OLIVARES, J. Comerciantes y políticos (Alicante 1875-1900), Alicante, 1987 , p. 125.

(3) A.C.C.S. sesión de 13 de junio 1886. 
como "cuerpos consultivos de la Administración pública". La normativa regula, asimismo, la constitución de las juntas directivas, el carácter de elector y elegible para las mismas, la elección de la presidencia y la financiación.

La Junta podría tener entre 10 y 40 vocales -en el caso de Sevilla se le adjudicaron 40-, de entre ellos se votaría una comisión directora formada por un presidente, dos vicepresidentes, contador, tesorero y secretario. La duración de la Junta sería de 6 años, pero con renovación trienal de la mitad de sus miembros; después de cada renovación serían votados nuevamente los citados cargos. Como organismo público se arbitraba su financiación en base a cuotas de hasta el $2 \%$ sobre la contribución que los comerciantes, industriales y navieros satisfacieran al Estado por sus actividades.

En conformidad con esto, en el caso sevillano tenemos la primera constitución de la Cámara el 1 de julio de 1912. En esa ocasión, la directiva fue integrada por:

Presidente: Hilario del Camino

Vicepresidente $1^{\mathrm{o}}$ : José $M^{\mathrm{a}}$ de Olmedo y Carranza

Vicepresidente $2^{\circ}$ : Luis Fernández-Palacios Labraña

Tesorero: Nicomedes García Barredo

Contador: Manuel Ramos Rejano

Secretario: Antonio González Ruiz (4)

El 31 de diciembre de 1914 acontece la correspondiente renovación trienal, que no conllevó, sin embargo, una importante modificación de la citada directiva: Romualdo Jiménez Cadenas pasa a desempeñar la vicepresidencia $1^{\underline{a}}$ y Dionisio García de la Mata, la tesorería (5). Para entonces, liberales y conservadores habían ido alcanzado una equilibrada representación en las sucesivas corporaciones. Desde 1916, sin embargo, será necesario tomar en consideración el progresivo peso dentro de la Cámara de una nueva entidad, la Unión Comercial.

\section{1917}

Abordamos ahora el análisis pormenorizado de la actuación de la Cámara en 1917; procederemos para ello, en primer lugar, a citar la distribución de sus cargos directivos y atenderemos después a las actividades y decisiones que se tomaron en este período, pero para

(4) A.C.C.S. sesión de 1 de julio 1912.

(5) A.C.C.S. sesión de 31 de diciembre 1914. 
comprender el alcance de las mismas retomaremos el aspecto de la composición y su evolución entre 1916 y 1918, con la intención de reflexionar sobre la coherencia de una línea concreta de intereses.

En enero de 1917 persistía en la presidencia de la Cámara Hilario del Camino y el resto de los cargos correspondían a: José de Pando, Luis Fernández-Palacios -vicepresidencias $1^{\circ}$ y $2^{\circ}$, respectivamente; Dionisio García de la Mata, contador, y Manuel Ramos Rejano, tesorero.

En 1917 se vivió en España la crisis política en medio del desorden y los graves perjuicios que tres años de guerra mundial habían acarreado a los sectores de la producción de bienes y su comercio. En Sevilla, esquemáticamente podría resumirse la situación en: las dificultades para el abastecimiento de materias primas y combustibles para la industria y el transporte; crisis para la exportación de algunos productos agrícolas tradicionales en el comercio sevillano y las tensiones sociales causadas por el encarecimiento de las subsistencias. En la Cámara se discutieron múltiples medidas y se solicitó al Gobierno la adopción de algunas soluciones. Esta institución canalizó su actividad fundamentalmente dentro del ámbito económico, permaneciendo formalmente al margen de los acontecimientos políticos. No obstante, se comprueba que esta actitud no fue compartida por la totalidad de sus miembros.

Al estudiar la actuación anual de este organismo, es posible aislar tres campos diferentes de ocupación: uno primero versado sobre problemas internos en el funcionamiento de las Cámaras; el segundo, la defensa de los intereses económicos de sus asociados y el tercero, las manifestaciones de carácter sociopolítico.

\subsection{Problemática de orden interno en el funcionamiento de las Cámaras}

La mayor dificultad se originaba en la indeterminación que acerca de la financiación derivaba de la ley de Bases de 1911. Otro aspecto tratado fue la formación de una Junta Consultiva Nacional para la Administración a partir de las Cámaras y también la adopción de una nuevo reglamento para el funcionamiento de estos organismos. Haremos una breve referencia a cada una de estas situaciones.

Según la citada ley de Bases, las Cámaras se financiarían con unas cuotas de sus asociados -todos los empresarios que por su actividad productiva pagaran al Estado una contribución de más de 40 pesetás- que podía alcanzar hasta el $2 \%$ de la contribución satisfecha al Estado. La 
percepción de esa cuota tenía que hacerse directamente por las Cámaras. La de Sevilla había fijado el $1 \%$, pero fue incapaz de conseguir su percepción generalizada entre los asociados. Por ello siempre cursó idéntica petición al Gobierno, que el Estado se encargara directamente del cobro del $2 \%$ y luego procediera a un reparto porcentual entre las cámaras, nunca fue atendida porque implicaría un recargo en los impuestos.

Dentro de este problema, la Cámara luchó en 1917 por hacer efectivo el cobro del 1\% que ciertas entidades financieras con sede central en Madrid, concretamente el Banco de España, el HispanoAmericano, el Crédit Lyonnais, y el Banco de Cartagena habían dejado de pagar desde 1912 con la argumentación de que hacían efectiva la cuota íntegra correspondiente a toda su actividad nacional a la Cámara de Madrid. Por otra parte, se tomó la decisión de elevar del 1 al $2 \%$ el porcentaje de la cuota.

La otra actividad en este ámbito corresponde a la formación dentro de la Dirección General de Comercio, Industria y Trabajo de una Junta Consultiva nacida de las Cámaras de Comercio, que estaría formada por siete presidentes de Cámaras, entre quienes aparecía el de Sevilla (6). La presencia sevillana está relacionada con la protesta conjunta de las dieciocho cámaras andaluzas ante el acaparamiento que las de Madrid y Barcelona hacían de todos los puestos de representación asignados a las cámaras en los organismos de la Administración (7).

Tras la inmediata constitución de la referida entidad, su primera decisión fue la reforma del reglamento de 29 de diciembre de 1911 por el que hasta entonces se regían las cámaras, el nuevo reglamento para aplicación de la ley de Bases entró en vigor por real decreto de 14 de marzo de 1918 (Gaceta del 15 de marzo) y, en conformidad con él, en octubre de ese año, 1918, se reconstituyó la Cámara de Sevilla.

\subsection{Actividades económicas y sociales}

Hacíamos antes un sintético resumen de las repercusiones del conflicto bélico mundial en la economía sevillana. En estrecha relación con ese panorama se inscribe la principal actividad de la Cámara: sus gestiones en favor de la mejora de los transportes y la defensa de la producción y el comercio del aceite e industrias relacionadas con este producto.

(6) Por el real decreto de 22 de 1917 formaban la Junta Basilio Paraíso (Cámara de Zaragoza), Horacio Echevarrieta (Bilbao, Diego Gómez (Sevilla), Camilo Pérez (Cartagena), Carlos Prast (Cámara de Industria de Madrid), Luis Ferrer (Barcelona) y Manuel Otero (Santiago).

(7) A.C.C.S. sesión 30 junio 1917. 
El problema de los transportes revestía una doble vertiente. Por un lado, la del transporte ferroviario, más duramente criticado y que provocó demandas más reivindicativas y, del otro, el transporte marítimo. La escasez de carbón y la mayor necesidad de tráfico ocasionó que la M.Z.A. careciera de locomotoras y vagones para conceder la necesaria fluidez al tráfico sevillano, lo que provocaba una inmediata repercusión en los abastecimientos de materias primas y en las necesidades de espacio para su comercio exterior. Las peticiones de remedio a la compañía y las denuncias al Gobierno fueron continuas.

Pero también la carestía y la insuficiencia del transporte marítimo afectaba a la actividad económica. Este asunto, no obstante, presentaba mayor complejidad, debido a la necesidad de conjugar dentro de la Cámara la protección de la producción y el comercio con el interés del poderoso sector naviero. Por ello, la publicación de un real decreto de Fomento, el 20 de octubre de 1917, sobre las nuevas tarifas para los fletes de cabotaje nacional, que contemplaba un fuerte incremento, se dirigía una circular al ministerio reconociendo la necesidad de revisar las tarifas, ante el encarecimiento de los combustibles y otros materiales necesarios para la navegación, pero no con una subida tan alarmante (8). Las reformas del puerto de Sevilla, que se hallaba en plena realización de las obras de la Corta de Tablada, la ampliación del muelle y el establecimiento del puente basculante de Alfonso XIII, y varias decisiones relacionadas con los seguros de navegación recabaron notable atención.

También fue continuo el apoyo que recibió de la Cámara la producción y el comercio de aceites y aceituna, el sector productivo más negativamente afectado en estos años de guerra por el control de su exportación y la dificultad para proveerse de los productos químicos necesarios para la elaboración. La misma complicación rodeaba a la elaboración de envases, por la falta de la hojalata necesaria (9). La vigilancia de la Cámara hacia cualquier aspecto que afectase, de un modo y otro, a este sector fue máxima y unánimes las demandas en favor de que se mejorasen las condiciones de exportación.

\subsection{Intervenciones de carácter sociopolítico}

La conflictividad política y social del año 1917 sólo nos ha dejado a través de la Cámara un pálido testimonio, en el que podemos distinguir

(8) 'A.C.C.S. sesión de 30 de noviembre de 1917.

(9) Se trató el abastecimiento de sosa y otros productos químicos, y las dificultades de la firma de envases García Longoria y Cía. 
dos aspectos: de un lado, su actividad con intermediaria entre la patronal y los obreros en un año crítico; de otro, la discusión sobre una más directa intervención en la conmoción política que se producía.

En el primer sentido, de los estudios sociales ya realizados sobre ese año, se deduce que en 1917 los conflictos que se registraron en Sevilla tuvieron su principal causa en los altos precios de los alimentos y, consecuentemente, se demandaba una subida en los salarios. La huelga general de agosto no fue secundada en Sevilla y sólo pararon los ferroviarios (10). En este marco, la Cámara jugó el papel de la concertación social y aconsejó a la patronal que aceptara subidas salariales y respetara las jornadas y condiciones de trabajo aprobadas (11). También levantó su voz para solicitar medidas de mayor control en el precio de los alimentos.

Por último, en el repaso de esta actividad, hay que indicar que no participó directamente en los acontecimientos políticos. Más aun, la Cámara de Sevilla mostró gran reticencia ante las reiteradas llamadas de otras Cámaras para que concediera su apoyo a la preparación de una asamblea general en la se analizaran los problemas nacionales; su respuesta a los llamamientos diversos fue siempre la misma: que se delimitara previamente la temática de la convocatoria y ella misma notificó sus intereses, confeccionando un listado que resume perfectamente el orden de sus preocupaciones y asume todos los aspectos tratados hasta ahora: 1 . subsistencias; 2 . aprovisionamiento de carbón; 3 . reorganización de los transportes; 4. que la economía nacional tomara medidas urgentes ante las repercusiones de la guerra; 5 . que se confeccionara una reglamentación que contemplara la periódica reunión nacional o regional de las cámaras (12). Es decir, que se trató de impedir el discurso político en un momento en el que se solicitaba la renovación democrática del sistema.

\section{EL PODER EN LA CAMARA}

El análisis de las principales actividades económicas desarrolladas en las primeras décadas del siglo revela que los mayores esfuerzos

(10) GONZALEZ FERNANDEZ, A. Lucha obrera en Sevilla. Conflictividad social, 19901917, pp. 119-120 y 190-191.

(11) Por ejemplo, en el mes de mayo, defendió el derecho al descanso dominical de los Dependientes de Comercio y en noviembre favoreció una subida del $25 \%$ en el salario de los obreros del muelle.

Similar actuación se ha señalado para las Cámaras de Madrid, BAHAMONDE MAGRO, A. y otros, La Cámara de Comercio e Industria de Madrid, 1887-1987. Historia de una institución centenaria, Madrid, 1988, pp. 205-206.

(12) A.C.C.S. sesión de 31 de diciembre de 1917. 
de la misma se dirigieron a la promoción de un sector concreto: la conjunción triangular entre productores de cereales y aceites, exportadores al por mayor y empresas navieras transportadoras.

Con este apoyo se ponía en práctica una de las dos funciones con la que se había nacido la institución, la defensa de sus asociados; si bien no de todos en igual manera. Mas, iniciábamos la comunicación subrayando la doble naturaleza en la que se descomponía la razón de ser de estas corporaciones, y nuevamente retomamos esa reflexión. Como organismos públicos, paralelos a la Administración, de carácter consultivo, las cámaras tenían impedido el ejercicio directo de actividad política, se insiste reiteradamente en ese carácter apolítico por definición.

Este condicionamiento se trasluce en la trayectoria de la Cámara de Sevilla desde su fundación hasta 1917 (13). Nos referimos, naturalmente, al ejercicio de una tarea política directa, ya que indirectamente, la Cámara colaboraba en la consolidación del orden canovista, conjugando, sobre la base de intereses económicos comunes, el cortés condominio entre liberales y conservadores, e incluso, la concurrencia del republicanismo. Es algo que se deja ver con un rápido vistazo a los hombres de su directiva: Hilario del Camino (conservador), José $M^{\mathbf{a}}$ de Olmedo y Carranza (liberal borbollista), José Montes Sierra (republicano)...

El apoliticismo declarado que subyace en ese compromiso entra en relación directa con el auge que a partir de 1914 adquiere una asociación patronal, la Unión Comercial, los grandes empresarios necesitaron ahora un instrumento más ágil, sin compromiso ni con la administración ni con el status quo de la Restauración. El período 19161918 podemos considerarlo como una etapa clave en la que se consuma la toma del control directo de la Cámara de Comercio por los hombres de la Unión Comercial. No hay duda de que la actitud conciliadora de la Cámara en materia social y su empeño en mantenerse al margen de la revisión política de la práctica canovista en 1917 reforzaron la tendencia.

En enero de 1917, como secuela de una discusión heredada de 1916, acerca del proyecto sobre exacción de arbitrios extraordinarios que el Ayuntamiento de Sevilla quería establecer con destino a un presupuesto extraordinario para las reformas urbanas, los hombre de la

(13) Este carácter constituye la tesis con que ha sido estudiada la trayectoria de la Cámara de Comercio e Industria de Madrid, BAHAMONDE MAGRO, A. y otros, ob. cit. p. 14 
Unión Comercial derribaron al sempiterno presidente de la Cámara, Hilario del Camino. El escrito de protesta que provocó la dimisión se hallaba firmado por: José de Pando y Fernández, Fernández-Palacios, Nicolás Luca de Tena, Enrique Martínez Lechón, Miguel García Longoria, Andrés Fernández Mensaque y Manuel Ramos Rejano, precisamente la directiva de la Unión Comercial. La presidencia recayó entonces en un hombre de la Unión, Diego Gómez Quintana.

Ese año introduce un impasse en el proceso, porque una real orden de 23 de octubre (Gaceta el 24 de octubre) aplazaba la renovación trienal en tanto no se dispusiera de nuevo reglamento. Ese momento no llegó a Sevilla hasta octubre de 1918 y entonces la Unión Comercial, impugnando los resultados del proceso electoral legal, colocó cerradamente en la directiva a sus afines: Nicolás Luca de Tena -que había quedado fuera de la Cámara en la elección- en la presidencia, Manuel Borrero Rebollo en la vicepresidencia $1^{\underline{a}}$ y Gabriel González Rivero, de tesorero; se mantuvieron Dionisio García de la Mata y Manuel Ramos Rejano, que eran de la Unión, ahora como vicepresidente $2^{\circ}$ y contador, respectivamente.

Tal homogenización se encuentra en la base del lanzamiento a la política activa de la Unión Comercial: ya es conocida su combatividad en 1918, que arrastró la caída de la Alcaldía liberal de Rodríguez de la Borbolla. Entre 1918 y 1931, desarrolló una papel político preeminente, en 1923 obtuvo un diputado en Cortes, Pedro Fernández-Palacios, su presidente (14).

En resumen, en la crisis final de la Restauración la actuación del empresariado sevillano se articulaba en dos instrumentos uno interior a la estructura administrativa del sistema y otro exterior, más combativo.

(14) ALVAREREY, L. La derecha en Sevilla durante la II República (1931-1936), tomó I pp. 75 y ss. Tesis Doctoral leída en la Universidad de Sevilla, 1990 (en prensa). 